\title{
SPECIAL INVOLUTIONS AND BULKY PARABOLIC SUBGROUPS IN FINITE COXETER GROUPS
}

\section{GÖTZ PFEIFFER ${ }^{\curvearrowleft}$ and GERHARD RÖHRLE}

\author{
(Received 20 May 2004; revised 20 September 2004)
}

Communicated by E. A. O'Brien

\begin{abstract}
The conjugacy classes of so-called special involutions parameterize the constituents of the action of a finite Coxeter group on the cohomology of the complement of its complexified hyperplane arrangement. In this note we give a short intrinsic characterisation of special involutions in terms of so-called bulky parabolic subgroups.
\end{abstract}

2000 Mathematics subject classification: primary 20F55; secondary 06A07.

\section{Introduction}

A finite Coxeter group $W$ with root system $\Phi$ spanning a Euclidean vector space $V$ acts on the space $V$ as a finite reflection group. Any involution in $W$ decomposes the space $V$ into the direct sum of a 1-eigenspace and a $(-1)$-eigenspace. With respect to such a decomposition, one might ask whether the projection of some root $\alpha \in \Phi$ onto an eigenspace is proportional to a root contained in the eigenspace or not. In general, such a projection need not be proportional to a root. An involution is called special if every root yields at least one projection which is proportional to a root. Special involutions have been introduced by Felder and Veselov [3] to describe the cohomology of the complement of the complexified hyperplane arrangement of $W$.

In this note, we give a short intrinsic characterisation of special involutions in terms of a property of parabolic subgroups. It is well known that the normalizer $N_{W}(P)$ of a parabolic subgroup $P$ of $W$ splits over $P$. Here we call the parabolic subgroup $P$ bulky, if the semidirect product $N_{W}(P)=P \rtimes N$ is in fact a direct product $P \times N$. We

(C) 2005 Australian Mathematical Society $1446-7887 / 05 \$ A 2.00+0.00$ 
show that an involution is special if and only if its corresponding parabolic subgroup is bulky; see Theorem 2.3.

In [3] Felder and Veselov considered the standard and twisted actions of a finite Coxeter group $W$ on the cohomology $H^{*}\left(\mathscr{M}_{W}\right)$ of the complement of the complexified hyperplane arrangement $\mathscr{M}_{W}$ of $W$. The twisted action is obtained by combining the standard action with complex conjugation; we refer the reader to [3] for precise statements. In a case by case argument, Felder and Veselov obtain a formula for all finite Coxeter groups $W$ for the standard action

$$
H^{*}\left(\mathscr{M}_{W}\right) \cong \sum_{\sigma \in X_{W}}\left(2 \cdot 1_{\langle\sigma\rangle}^{W}-\varrho\right)
$$

as $\mathbb{C} W$-modules, where $X_{W}$ is a set of representatives of the $W$-conjugacy classes of the special involutions in $W, \varrho$ is the regular representation of $W$, and $1_{\langle\sigma\rangle}^{W}$ is the $\mathbb{C} W$-module induced from the trivial $\mathbb{C}\langle\sigma\rangle$-module. In case $W$ is crystallographic, this formula can be deduced from earlier work of Lehrer [8,9] and FleischmannJaniszczak [4,5]. The new aspect of [3] in the theory is a uniform geometric description of the sets $X_{W}$ of $W$-conjugacy classes of special involutions used in the formula above.

Felder and Veselov give a similar formula for the twisted action where the summation is taken over the set of even elements from $X_{W}$.

\section{Notation and preliminaries}

Throughout, $W$ denotes a finite Coxeter group, generated by a set of simple reflections $S \subseteq W$; see [1] or [6] for a general introduction into the theory of Coxeter groups. For $J \subseteq S$, let $W$, be the parabolic subgroup of $W$ generated by $J$ and denote by $w_{J}$ the unique word in $W_{J}$ of maximal length (with respect to $S$ ). Let $T=S^{W}$ be the set of all reflections of $W$. Let $\Phi$ be a root system with Coxeter group $W$ and $\Phi_{J}$ the root subsystem of $\Phi$ corresponding to $W_{J}$. Set $V:=\mathbb{Z} \Phi \otimes_{\mathbb{Z}} \mathbb{R}$. Then $V$ affords the usual reflection representation of $W$. For each involution $\sigma \in W$ we have a direct sum decomposition $V=V_{1} \oplus V_{-1}$, where $V_{1}$ and $V_{-1}$ are the 1 and (-1)-eigenspaces of $V$ of $\sigma$, respectively. For $\epsilon= \pm 1$ let $\Phi_{\epsilon}:=\Phi \cap V_{\epsilon}$. Note that for $\sigma=w_{J}$ we have $\Phi_{-1}=\Phi_{J}$. Following [3], we say that an involution $\sigma$ in $W$ is special, if for any root $\alpha \in \Phi$ at least one of its projections onto $V_{\epsilon}$ is proportional to a root in $\Phi_{\epsilon}$. Clearly, this definition does not depend on the choice of root system for $W$.

The conjugacy classes of involutions in $W$ have been classified by Richardson [10, Theorem A] and Springer [11] in terms of the parabolic subgroups of $W$ whose longest element is central. More precisely, each involution is conjugate to a longest element $w_{J}$ which is central in $W_{J}$ for some $J \subseteq S$.

The normalisers of parabolic subgroups of finite Coxeter groups have been described by Howlett [7] and Brink and Howlett [2]. Accordingly, the normaliser 
$N_{W}\left(W_{J}\right)$ of $W_{J}$ in $W$ is a semi-direct product of the form $W_{J} \rtimes N_{J}$, where $N_{J}$ is itself a semi-direct product of a Coxeter group of known type and a group $M_{J}$, [7, Corollary 7]. It turns out, however, that in the case when $w_{J}$ is central in $W_{J}$ the group $M_{J}$ is trivial.

Proposition 2.1. $M_{J}$ acts faithfully as inner graph automorphisms on $W_{J}$. In particular, if $w_{J}$ is central in $W_{J}$, then $M_{J}=\{1\}$.

Proof. According to the tables in [2], if $W$ is irreducible and $J$ is such that $|S \backslash J|=2$, then a non-trivial generator of the group $M_{J}$ exists only when either $W$ is of type $E_{7}$ and $W_{J}$ is of type $A_{4} \times A_{1}$, or $W$ is of type $D_{2 n}$ and $W_{J}$ is of type $A_{2 n-2}$ or $A_{2 k} \times A_{2 l}$ with $k \neq l$ and $k+l=n-1$. Let us say that $W_{J}$ is an M-parabolic subgroup of $W$ in such a case. An easy check shows that if $W_{J}$ is an M-parabolic subgroup of $W$, then $M_{J}$ induces the same non-trivial graph automorphism on $W_{J}$ as conjugation by $w_{J}$. In general, it follows from [2] that $M_{J}$ is trivial unless a conjugate $L$ of $J$ lies in a subset $K \subseteq S$ such that $|K \backslash L|=2$ and $W_{K}=W_{N} \times W_{K^{\prime}}$ and $W_{L}=W_{N} \times W_{L^{\prime}}$ for suitable subsets $K^{\prime}, L^{\prime}, N \subseteq S$ and $W_{L^{\prime}}$ is an M-parabolic subgroup of $W_{K^{\prime}}$. Now $M_{L}$ induces a non-trivial inner automorphism on $W_{L}$ and so does $M_{J}$ on $W_{J}$.

By [7, Corollary 9], $M_{J}$ intersects the centraliser of $J$ in $N_{J}$ trivially, and hence acts faithfully on $W_{J}$.

The centraliser of the involution $w_{J}$ and the normaliser of the parabolic subgroup $W_{J}$ of $W$ coincide; see [3, Proposition 7]. We give a new proof of this property.

PROPOSITION 2.2. For each $J \subseteq S$ the element $w_{J}$ is central in $W_{J}$ if and only if $C_{W}\left(w_{J}\right)=N_{W}\left(W_{J}\right)$.

PRoOF. Suppose $w_{J}$ is central in $W_{J}$. Then $W_{J} \subseteq C_{W}\left(w_{J}\right) \cap N_{W}\left(W_{J}\right)$. To show that $C_{W}\left(w_{J}\right)=N_{W}\left(W_{J}\right)$ it thus suffices to consider the set $D_{J}=\{x \in W: l(s x)>$ $l(x)$ and $l(x s)>l(x)$ for all $s \in J\}$ of distinguished double coset representatives of $W_{J}$ in $W$.

We have $l\left(w^{x}\right)=l(w)$ for all $w \in W_{J}, x \in N_{J}=\left\{x \in D J: J^{x}=J\right\}$. In particular, $w_{J}^{x}=w_{J}$ for $x \in N_{J}$. Hence $N_{W}\left(W_{J}\right) \subseteq C_{W}\left(w_{J}\right)$.

Conversely, let $x \in C_{W}\left(w_{J}\right) \cap D_{J}$. Then $w_{J} \in W_{J} \cap W_{J}^{x}=W_{J \cap J x}$; see, for example, [6, (2.1.12)]. It follows that $J=J^{x}$ whence $C_{W}\left(w_{J}\right) \subseteq N_{W}\left(W_{J}\right)$.

We call the parabolic subgroup $W_{J}$ bulky (in $W$ ) if $N_{W}\left(W_{J}\right)=W_{J} \times N_{J}$, that is, if $N_{J}$ acts trivially on $W_{J}$. The main result of this note is the following theorem.

THEOREM 2.3. Let $J \subseteq S$ be such that $w_{J}$ is central in $W_{J}$. Then the involution $w_{J}$ is special if and only if $W_{J}$ is bulky in $W$. 
In our arguments we do make use of the classification of the irreducible Coxeter groups and the structure of the root systems of Weyl groups. We also use the notation and labelling of the Dynkin diagram of $W$ as in [1, Planches I-IX].

\section{Special involutions and bulky parabolic subgroups}

We maintain the notation from the previous sections.

LEMMA 3.1. If $\operatorname{dim} V_{1}=1$ and $\Phi_{1} \neq \varnothing$ or if $\operatorname{dim} V_{-1}=1$, then $w_{J}$ is special. In particular, $\pm \sigma$ is special for every reflection $\sigma \in T$.

PROOF. The projection of any root onto a one-dimensional space generated by a root $\alpha$ is clearly proportional to $\alpha$.

REMARK 3.2. The element $w_{J}$ is central in $W_{J}$ if and only if $W_{J}$ has no components of type $A_{n}$ with $n \geq 2$, of type $D_{2 n+1}$ with $n \geq 2$, of type $E_{6}$, or of type $I_{2}(2 m+1)$, $m \geq 2$; see $[10,1.12]$.

PROOF OF THEOREM 2.3. We may assume that $W$ is irreducible. By [2, Theorem B] and our Proposition 2.1 the group $N_{J}$ is generated by certain conjugates of elements of the form $w_{L} w_{K}$, where $L \subseteq K \subseteq S$ such that $L$ is a conjugate of $J,|K \backslash L|=1$ and $L^{w_{K}}=L$. If $s^{w_{L} w_{K}}=s$ for all $s \in L$, then $w_{L} w_{K}$ centralises $W_{L}$ and so its conjugate centralises $W_{J}$. Obviously, $s^{w_{L} w_{K}}=s^{w_{K}}$ for all $s \in L$, since $w_{L}$ is central in $W_{L}$.

Now suppose that $W_{J}$ is not bulky in $W$, that is, $N_{J}$ does not centralise $W_{J}$. Then there exists a conjugate $L$ of $J$ and a subset $K \subseteq S$ such that $L \subseteq K$ with $|K \backslash L|=1$ and $w_{K}$ induces a non-trivial graph automorphism on $W_{L}$. It follows that $W_{K}=W_{N} \times W_{K^{\prime}}$ for suitable $N, K^{\prime} \subseteq S$ where the type of $W_{K^{\prime}}$ is one of those listed in Remark 3.2. Since $w_{L}$ is central in $W_{L}$, it follows that $W_{L}=W_{N} \times W_{L^{\prime}}$ where $W_{L^{\prime}}$ is a product of components of types not listed in Remark 3.2. Inspection of the maximal parabolic subgroups of $W_{K^{\prime}}$ shows that $W_{L^{\prime}}$ is of type $D_{2 n}$ and $W_{K^{\prime}}$ is of type $D_{2 n+1}, n \geq 1$; this includes the case where $A_{1}^{2}$ embeds into $A_{3}$ for $n=1$.

Without loss of generality we may assume $N=\varnothing, K^{\prime}=K=S$ and $L^{\prime}=L=J$. So let

$$
\Phi=\left\{ \pm \varepsilon_{i} \pm \varepsilon_{j}: 1 \leq i<j \leq 2 n+1\right\}
$$

be a root system of type $D_{2 n+1}$ and consider the simple root $\alpha=\varepsilon_{1}-\varepsilon_{2}$. It is easy to check that $\left\{\varepsilon_{2}, \ldots, \varepsilon_{2 n+1}\right\}$ is a basis of $V_{-1}$ and that $V_{1}$ is the $\mathbb{R}$-span of $\varepsilon_{1}$. Obviously $V_{1}$ contains no root, hence $\Phi_{1}=\Phi \cap V_{1}=\varnothing$. Consequently, no projection of a root in $\Phi$ on $V_{1}$ is proportional to a root in $\Phi_{1}$. Next we show that the projection of $\alpha$ 
onto $V_{-1}$ is not proportional to any root in $\Phi_{-1}=\Phi \cap V_{-1}$. Recall that $\Phi_{-1}=\Phi_{J}$, a root system of type $D_{2 n}$ consisting of the roots $\pm \varepsilon_{i} \pm \varepsilon_{j}$, with $2 \leq i<j \leq 2 n+1$. The projection of $v \in V$ onto $V_{-1}$ is given by $\frac{1}{2}\left(v-w_{J}(v)\right)$. Hence $\alpha$ projects onto $\frac{1}{2}\left(\alpha-w_{J}(\alpha)\right)=-\varepsilon_{2}$ and therefore is not proportional to any root in $\Phi_{-1}$. Thus $w_{J}$ is not special, as required.

For the converse, suppose that $W_{J}$ is bulky in $W$, that is, $N_{J}$ acts trivially on $W_{J}$. If $J=\varnothing$ or $J=S$, then clearly $w_{J}$ is a special involution. So let us assume that $J \neq \varnothing, S$. We consider the different types of irreducible Coxeter groups in turn.

If $W$ is of type $A_{n}(n \geq 1)$, then, by Remark 3.2, $W_{J}$ is necessarily a direct product of components of type $A_{1}$. But if there is more than one such component, $N_{J}$ permutes them non-trivially. Hence $W_{J}$ is of type $A_{1}$ and the claim follows by Lemma 3.1.

If $W$ is of type $C_{n}(n \geq 2)$, then, by Remark 3.2, $W_{J}$ is a direct product of a component of type $C_{m}, 0 \leq m<n$ and further components of type $A_{1}$. As before there cannot be more than one component of type $A_{1}$. Hence $W_{J}$ is of type $C_{m}$ or of type $C_{m} \times A_{1}$ for some $m<n$. In any case, $W_{J}$ has a component of type $C_{m}$.

Let

$$
\Phi=\left\{ \pm 2 \varepsilon_{i}: 1 \leq i \leq n\right\} \cup\left\{ \pm \varepsilon_{i} \pm \varepsilon_{j}: 1 \leq i<j \leq n\right\}
$$

be the root system of type $C_{n}$. Consider the maximal rank subsystem $\Phi^{\prime}$ of type $C_{m} \times C_{n-m}$ consisting of the long roots $\left\{ \pm 2 \varepsilon_{i}: 1 \leq i \leq n\right\}$ and the short roots $\left\{ \pm \varepsilon_{i} \pm \varepsilon_{j}: 1 \leq i<j \leq m\right.$ or $\left.m+1 \leq i<j \leq n\right\}$. Let $U_{1}$ be the subspace of $V$ spanned by $\varepsilon_{1}, \ldots, \varepsilon_{m}$ and $U_{2}$ the subspace spanned by $\varepsilon_{m+1}, \ldots, \varepsilon_{n}$. Then $U_{2} \cap \Phi$ is a root system of type $C_{n-m}$. All the long roots $\pm 2 \varepsilon_{i}$ of $\Phi$ are contained in $\Phi^{\prime}$. A short root $\pm \varepsilon_{i} \pm \varepsilon_{j}$ is either contained in $\Phi^{\prime}$ or both its projections on $U_{1}$ and $U_{2}$ are proportional to a root in $\Phi^{\prime}$. By construction, the $(-1)$-eigenspace $V_{-1}$ of $w_{J}$ contains $U_{1}$. Hence every root that lies in $U_{1}$ or is proportional to a root in $U_{1}$ is also proportional to a root in $V_{-1}$. It remains to consider the roots in $U_{2}$. Without loss of generality we can now assume that $m=0$. Then $W_{J}$ is of type $A_{1}$ and the claim follows by Lemma 3.1.

If $W$ is of type $D_{2 n+1}(n \geq 2)$, then, by Remark $3.2, W_{J}$ is a direct product of an optional component of type $D_{2 m}, 1 \leq m \leq n$ and further components of type $A_{1}$. As before there cannot be more than one component of type $A_{1}$. And $N_{J}$ acts non-trivially on a component of type $D_{2 m}$. Hence $W_{J}$ is of type $A_{1}$ and the claim follows by Lemma 3.1 .

If $W$ is of type $D_{2 n}(n \geq 2)$, then, by Remark $3.2, W_{J}$ is a direct product of an optional component of type $D_{2 m}, 1 \leq m<n$ and further components of type $A_{1}$. As before there cannot be more than one component of type $A_{1}$. The non-trivial action of the parabolic subgroup of type $D_{2 m+1}$ on a component of type $D_{2 m}$ then restricts the type of $W_{J}$ to either $A_{1}$ or $D_{2(n-1)} \times A_{1}$. In the latter case, $V_{1} \cap \Phi$ is a root system of type $A_{1}$ and so in both cases the claim follows by Lemma 3.1. 
If $W$ is of type $I_{2}(m)(m \geq 5)$, then $W_{J}$ is of type $A_{1}$ and the claim follows by Lemma 3.1.

Finally, if $W$ has type $E_{6}, E_{7}, E_{8}, F_{4}, H_{3}$, or $H_{4}$, then the claim is established by inspection.

REMARK 3.3. Felder and Veselov prove one implication of Theorem 2.3, namely that $W_{J}$ is bulky if $w_{J}$ is special in a case by case analysis [3, Proposition 10].

REMARK 3.4. Bulky parabolic subgroups can be easily classified. It turns out that if $W$ has a central longest element, then $w_{J}$ is central in $W_{J}$ whenever $W_{J}$ is bulky. Otherwise, $W$ has bulky parabolic subgroups $W_{J}$ which are not associated with a conjugacy class of involutions in $W$.

\section{Acknowledgements}

This paper was written while the first author was visiting the School of Mathematics of the University of Birmingham under a Scheme 2 LMS grant. We are grateful to the LMS for its financial support and to the members of the School for their hospitality. We also wish to thank A. Borovik for bringing the problem of an intrinsic characterisation of special involutions to our attention.

\section{References}

[1] N. Bourbaki, Groupes et algèbres de Lie. Chapitres IV-VI (Hermann, Paris, 1968).

[2] B. Brink and R. B. Howlett, 'Normalizers of parabolic subgroups in Coxeter groups', Invent. Math. 136 (1999), 323-351.

[3] G. Felder and A. P. Veselov, 'Coxeter group actions on the complement of hyperplanes and special involutions', J. Eur. Math. Soc. (JEMS) 7 (2005), 101-116.

[4] P. Fleischmann and I. Janiszczak, 'The lattices and Möbius functions of stable closed subrootsystems and hyperplane complements for classical Weyl groups', Manuscripta Math. 72 (1991), $375-403$.

[5] - Combinatorics and Poincaré polynomials of hyperplane complements for exceptional Weyl groups', J. Combin. Theory Ser. A 63 (1993), 257-274.

[6] M. Geck and G. Pfeiffer, Characters of finite Coxeter groups and Iwahori-Hecke algebras, London Math. Soc. Monogr. New Series 21 (Oxford University Press, New York, 2000).

[7] R. B. Howlett, 'Normalizers of parabolic subgroups of reflection groups', J. London Math. Soc. (2) 21 (1980), 62-80.

[8] G. I. Lehrer, 'On hyperoctahedral hyperplane complements', in: The Arcata Conference on Representations of Finite Groups (Arcata, CA, 1986), Proc. Sympos. Pure Math. 47 (Amer. Math. Soc., Providence, RI, 1987) pp. 219-234.

[9] —_ 'On the Poincaré series associated with Coxeter group actions on complements of hyperplanes', J. London Math. Soc. (2) 36 (1987), 275-294. 
[10] R. W. Richardson, 'Conjugacy classes of involutions in Coxeter groups', Bull. Austral. Math. Soc. 26 (1982), 1-15.

[11] T. A. Springer, 'Some remarks on involutions in Coxeter groups', Comm. Algebra 10 (1982), 631-636.

Department of Mathematics

National University of Ireland

Galway

Ireland

e-mail: goetz.pfeiffer@nuigalway.ie
School of Mathematics University of Birmingham

Birmingham B15 2TT

United Kingdom

e-mail: ger@for.mat.bham.ac.uk 